\title{
Correction to: Imaging the myocardial ischemic cascade
}

\author{
Arthur E. Stillman ${ }^{1}$ (D) Matthijs Oudkerk ${ }^{2}$. David A. Bluemke ${ }^{3} \cdot$ Menko Jan de Boer $^{4} \cdot$ Jens Bremerich ${ }^{5}$. \\ Ernest V. Garcia ${ }^{1} \cdot$ Matthias Gutberlet $^{6} \cdot$ Pim van der Harst ${ }^{7} \cdot$ W. Gregory Hundley ${ }^{8} \cdot$ Michael Jerosch-Herold $^{9}$. \\ Dirkjan Kuijpers $^{10}$ - Raymond Y. Kwong ${ }^{11}$. Eike Nagel ${ }^{12}$. Stamatios Lerakis ${ }^{13}$. John Oshinski ${ }^{1}$. Jean-François Paul ${ }^{14}$. \\ Riemer H. J. A. Slart ${ }^{15} \cdot$ Vinod Thourani $^{16} \cdot$ Rozemarijn Vliegenthart $^{17} \cdot$ Bernd J. Wintersperger $^{18}$
}

Published online: 24 July 2018

(c) Springer Nature B.V. 2018

\section{Correction to: \\ The International Journal of Cardiovascular Imaging \\ https://doi.org/10.1007/s10554-018-1330-4}

In the original publication of the article, the seventh author name "Matthias Gutberlet" has been misspelt. The correct name is given in this correction. The original version of this article was revised.

The original article can be found online at https://doi.org/10.1007/ s10554-018-1330-4.

Arthur E. Stillman aestill@emory.edu

1 Department of Radiology and Imaging Sciences, Emory University, 1365 Clifton Rd NE, Atlanta, GA 30322, USA

2 Center of Medical Imaging, University Medical Center Groningen, Groningen, The Netherlands

3 Department of Radiology and Imaging Sciences, National Institute of Biomedical Imaging and Bioengineering, Bethesda, MD, USA

4 Department of Cardiology, Radboud University Medical Center Nijmegen, Nijmegen, The Netherlands

5 Department of Radiology, University of Basel Hospital, Basel, Switzerland

6 Diagnostic and Interventional Radiology, University Hospital Leipzig, Leipzig, Germany

7 Department of Genetics, University Medical Center Groningen, Groningen, The Netherlands

8 Departments of Internal Medicine \& Radiology, Wake Forest University, Winston-Salem, NC, USA

9 Department of Radiology, Brigham and Women's Hospital, Boston, MA, USA
10 Department of Radiology, Haaglanden Medical Center, The Hague, The Netherlands

11 Department of Cardiology, Brigham and Women's Hospital, Boston, MA, USA

12 Institute for Experimental and Translational Cardiovascular Imaging, DZHK Centre for Cardiovascular Imaging, University Hospital, Frankfurt/Main, Germany

13 Department of Medicine, Emory University, Atlanta, GA, USA

14 Department of Radiology, Institut Mutualiste Montsouris, Paris, France

15 Department of Nuclear Medicine and Molecular Imaging, University of Groningen, University Medical Center Groningen, Groningen, The Netherlands

16 Department of Cardiac Surgery, MedStar Heart and Vascular Institute, Georgetown University, Washington, DC, USA

17 Department of Radiology, University Medical Center Groningen, Groningen, The Netherlands

18 Department of Medical Imaging, University of Toronto, Toronto, Canada 